\section{Atopisches Ekzem: Was ist gesichert?}

\section{Ungeachtet der noch anhaltenden Diskussionen um die pathophysiologischen Grundlagen eines atopischen Ekzems gibt es handfeste Empfehlungen für die Therapie - sowohl im Akutstadium wie auch für der Dauertherapie. Vor allem muß der Patient aber lernen, mit seiner Krankheit eigenverantwortlich umzugehen, lernen, „sein eigener Hautarzt zu werden“.}

Das atopische Ekzem (AE) gehört zum atopischen Formenkreis und ist untrennbar verbunden mit Asthma bronchiale und der allergischen Rhinokonjunktivitis. Allerdings, so J. Ring (München), ist die Pathophysiologie dieser Krankheit noch verwirrender als beim Asthma: Zum einen beeinflussen klassische allergisch-entzündliche immunologische Vorgänge das klinische Bild, nicht vergessen werden dürfen aber auch Barrierefunktionsstörungen durch die Hautbeschaffenheit der Patienten, eine mikrobielle Kolonisation sowie psychosomatische Einflüsse.

Auch beim atopischen Ekzem: intrinsische und extrinsische Form Wie beim Asthma muß auch beim AE eine extrinsische von einer intrinsischen Form (nach Wüthrich) unterschieden werden. Die intrinsische Form manifestiert sich klinisch identisch, ist aber nicht IgE-vermittelt. Auch weiß man heute über die Existenz von Auto-Antikörpern, die für besonders schwere Verläufe eines atopischen Ekzems verantwortlich sind so die ersten Ergebnisse der Arbeitsgruppen um Valenta, Stingl und Ring.

Als pathophysiologisches Modell für die Entwicklung des AE schlug Ring folgendes Konzept vor: „Es startet als TH2-Reaktion und geht in der chronischen Phase in eine TH1-Reaktion über - weshalb das klinische Bild in dieser Phase dem eines chronischen Kontaktekzems ähnelt. Bei der schweren perpetuierenden AE-Form stehen Autoimmunprozesse im Vordergrund.“

Die klinische Diagnose des AE be-

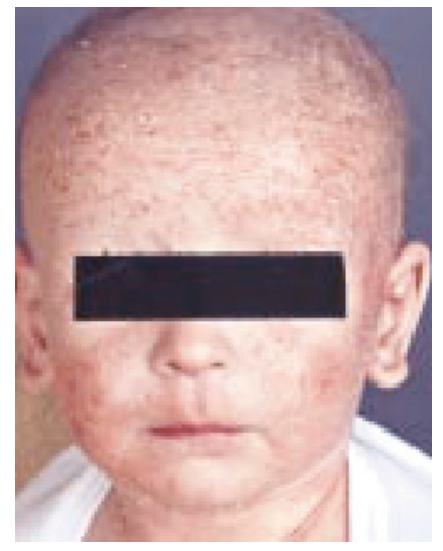

Akutes atopisches Ekzem mit Befall vor krusteten, nässenden Rötungen der Haut.

ruht in erster Linie auf der Art der Hautveränderungen und deren Lokalisation sowie auf anamnestischen Erhebungen - vor allem Juckreiz sowie atopische Eigen- und Fremdanamnese. In der Allergiediagnostik, so Ring, kann der Atopie-Patch-Test wertvolle Hinweise liefern.

\section{Therapie: Defizite der} trockenen Haut ausgleichen

Bei der Therapie des AE darf die Pathophysiologie der Haut nicht vergessen werden. Die Kranken haben eine „trockene Haut": Diese bildet weniger epidermale Lipide, verliert als Zeichen einer Barrierestörung transepidermal vermehrt Wasser und hat eine veränderte Oberfläche, die die Patienten als „rauh“ empfinden. Diesen Veränderungen, so Ring, kommt in der Pathophysiologie des AE eine den immunologischen Veränderungen vergleichbare allem des Gesichts mit großflächigen ver-
Bedeutung $\mathrm{zu}$, ist aber weit weniger gut erforscht.

Angezeigt als Basistherapie sind deshalb spreitende Ölbäder, die einen Lipidfilm auf der Haut zurücklassen und leichte, die Haut nicht reizende Kleidung. Als Karenzmaßnahmen empfiehlt sich das Meiden von Allergenen soweit relevant. Zum Schutz vor allem vor nächtlichen Kratzanfällen können Verbände eingesetzt werden, das Festbinden der Kinder gilt als obsolet. Bei unerträglichem Juckreiz empfiehlt sich auch die Anwendung des „Nassen Schlafanzugs“" oder einer kühlenden Lotion.

\section{Duschen und Baden \\ nicht unbedingt einschränken}

Duschen und Waschen müssen bei guter Rückfettung nicht verboten werden, „schließlich werden so schädliche Bakterien und Allergene von der Haut eliminiert", sagte Ring. Bei therapeutischen Versuchen mit UVA1-Licht ist man aber speziell bei Kindern wegen der unbekannten Spätrisiken zurückhaltend.

Im Akutstadium sind Kortikoide indiziert, sie drängen die Entzündung rasch zurück. Als Alternativen stehen extern Bufexamac - cave Kontaktallergien (!) - und zumindest bei kleinen Kindern Ammoniumbituminosulfonat (aus Schieferöl) zur Verfügung. Für die Juckreizlinderung haben sich Polidocanol und Antihistaminika bewährt. Im Bedarfsfall dürfen Antiseptika (extern) und Antibiotika (systemisch) nicht vergessen werden. Eine Hyposensibilisierung ist in der Regel nicht anzuraten. Immunsuppressiva bleiben den schweren Formen des AE vorbehalten.

„Das wichtigste ist aber, daß der Patient lernt, mit seiner Krankheit umzugehen - und die Familie muß sich mit Wohnungsausstattung, Essen oder Urlaubsplanung darauf einrichten." Das von Ring vertretene Konzept des „Patienten-Management" zusammengefaßt: „Werden Sie Ihr eigener Hautarzt, der weiß, wann er welche Creme auf welche Körperpartie aufzutragen hat. Für Störfälle sind wir Ärzte weiterhin für Sie zuständig.“

J. Ring (München) anläßlich der 21. Tagung der Deutschen Gesellschaft für Allergologie und klinische Immunologie, München 1999. 